\title{
Consideration of Social Aspects and Mental Health of Community-Dwelling Elderly People: A Literature Review
}

\author{
Junko Imaiso \\ Kansai University of Social Welfare, Ako-shi, Hyogo-Pref, Japan \\ Email: imaiso@kusw.ac.jp
}

How to cite this paper: Imaiso, J. (2020) Consideration of Social Aspects and Mental Health of Community-Dwelling Elderly People: A Literature Review. Health, 12, 486-498.

https://doi.org/10.4236/health.2020.125038

Received: April 16, 2020

Accepted: May 24, 2020

Published: May 27, 2020

Copyright ( 2020 by author(s) and Scientific Research Publishing Inc. This work is licensed under the Creative Commons Attribution-NonCommercial International License (CC BY-NC 4.0). http://creativecommons.org/licenses/by-nc/4.0/ (c) (i) (8) Open Access

\begin{abstract}
Purpose: A literature review was conducted to explore the social factors and mental health-related scales, and to examine associations between social factors and mental health of the elderly, in order to consider the social aspects and mental health as outcome measurements for an effective community-based care. Methods: An electronic search for English articles published in peer-reviewed journals up to October 2019 was performed using PubMed, MEDLINE, and CINAHL with the following search terms: "social cohesion," "mental health," "elderly," and "community." Inclusion criteria were 3 as follows; 1) studies in community-dwelling elderly people or participants including elderly individuals; 2) studies with clear descriptions of social factors in the Methods section; and 3) studies with clear descriptions of health-related items (e.g., mental health) in the Methods section. The extracted articles were subjected to narrative review. Results: In total, 118 articles were identified, of which 11 met the inclusion criteria after critical review of the full text. 6 mental health-related scales were perceived in order to assess of mental health or depression or anxiety of the elderly. And, 4 factors related to social aspects; neighborhood social cohesion, social capital, neighborhood environment, and social support, were perceived. Significant associations between social aspects and mental health of the elderly were examined. In social cohesion, 6 studies reported a significant association with elderly mental health. In social capital, 3 studies reported a significant association with elderly mental health. In neighborhood environment, 3 studies reported a significant association with elderly mental health. Discussion: In the development of an effective community-based care, it may be expected to be focused on social aspects to lead to promoting mental health of the elderly. For the further investigation, it will need to be considered more clearly defined measurements related to social aspects and mental health of the elderly,
\end{abstract}


and it will be more important to pile the research data globally.

\section{Keywords}

Aging, Social Factor, Mental Health, Community-Based, Population Health

\section{Introduction}

Aging and super-aging of society have become global concerns. As changes in physical and mental health often accompany aging, progressive aging is a global human health issue. Effective community care should thus be considered globally to gain insight into the prevention of caregiving for elderly people and the promotion of seamless care in medicine, prevention, and welfare. Innovation of community-based care is expected to improve not only the quality of life (QOL) of community-dwelling elderly people but also lead to a better community environment that contributes to healthy living among the younger generations, such as children and the working population. In this context, considering the health of the whole population by promoting health among the elderly will become increasingly relevant.

Each country is facing challenges specific to their own health policies and social changes. In Japan, the Draft Act on Amendatory Law to the Related Acts for Securing Comprehensive Medical and Long-Term Care in the Community came into effect in 2014 [1]. The Japanese Ministry of Health, Labour and Welfare proposed that an integrated community-based care system be established in each municipality by 2025 , considering each community characteristic. This is expected to lead to the development of a truly effective, sustainable, and integrated community-based care system which takes into consideration the characteristics of community and promotes healthy living of community residents. However, the evidence of effective integrated community-based care appeared not to be enough worldwide. Evaluation items and outcomes of integrated community-based care need to be clarified and related findings are accumulated, considering the characteristics of community.

According to a previous literature review, 3 aspects of healthy living among community-dwelling elderly people can be considered outcomes of effective integrated community-based care: physical and social functioning, physical and mental health, and QOL [2]. In particular, it was suggested that social aspects need to be more focused on, considering the character of community environments such as urban or rural.

In additions, focusing on differences in environment between urban and rural communities as one of the characteristics of residential community, it has been suggested that social function and mental health or depression of elderly individuals might be more important as outcome measures of effective integrated community-based care [3]. And, it may be important for us to understand the 
associations between the defined social factors and mental health of community-dwelling elderly people, in order to consider the outcomes in the development of a truly effective, sustainable, and integrated community-based care system from the perspective of the community characteristics.

Various measurement tools are used to assess social aspects, as well as mental health. The social factors in a research need to be identified. In social aspects, focusing on social cohesion has been suggested as a way to gain a deeper understanding of social resilience, as well as for designing interventions for more sustainable and resilient systems [4].

Social cohesion may become one of the important social factors when considering how to further develop an effective and sustainable integrated community-based care system according to community characteristics, while taking into consideration social factors and the mental health of elderly people.

The purpose of this study was to explore the social factors and mental health-related scales, and to examine the associations between the social factors and mental health through a literature review, in order to consider the social aspects and mental health of the elderly as outcome measurements for an effective community-based care.

\section{Methods}

Electronic searches for English articles published in peer-reviewed journals up to October 2019 were conducted within PubMed, MEDLINE, and CINAHL, employing the following search terms: "social cohesion," "mental health," "elderly," and "community."

Identified articles were screened by title and abstract, and selected articles were subjected to full-text assessment and critical review according to the following 3 inclusion criteria: 1) studies conducted in community-dwelling elderly people or community-dwelling people including elderly people as participants; 2) studies with clear descriptions of social factors in the Methods section; 3) studies with clear descriptions of health-related items (e.g., mental health) in the Methods section. A narrative review was conducted in accordance with the present study objective. Same mental health-related scales used in each article were collected, and items with a similar meaning with regard to social function were assessed by collecting corresponding information from each article and perceived the social factors of community-dwelling elderly people. In addition, similar measures regarding social functions and mental health were assessed by collecting corresponding information from each article. Then, the associations between social factors and mental health of community-dwelling elderly people were examined.

\section{Results}

In total, 118 articles were identified by the electronic search. After excluding 78 articles on the basis of title and abstract, 40 were selected, of which 11 met the 
inclusion criteria after full-text review; these were included in the final analysis (Figure 1).

\section{Outline of the Research Articles}

Table 1 summarizes data extracted from the 11 articles: 5 were published in 2018 [5] [6] [7] [8] [9], 2 were published in 2017 [10] [11], and 1 each was published in 2015 [12], 2014 [13], 2013 [14] and 2011 [15]. 6 of these studies were conducted in the United States [5] [6] [7] [9] [10] [11], 2 in the United Kingdom [13] [15], 2 in Japan [8] [14], and 1 in Taiwan [12]. The number of community-dwelling older people enrolled as participants in the 11 studies ranged from 149 to $19,656.6$ studies [5] [6] [8] [12] [14] [15] targeted individuals aged $\geq 65$ years, 3 [7] [10] [11] targeted those aged $\geq 60$ years, and 1 [9] targeted those aged $\geq 57$ years; $1[13]$ included participants aged $\geq 65$ years in the study sample.

\section{Mental Health-related Scales}

6 mental health-related scales were used in the 11 studies: Warwick-Edinburgh Mental Wellbeing Scale (WEMWBS) with 14 positively-worded statements [13] [15]; 15-item Geriatric Depression Scale (GDS-15) [8] [12] [14]; Center for Epidemiologic Studies Depression Scale (CES-D) [5] [9] [11]; Depression Scale-Anxiety (HADS-A) with 7 symptoms [7] [10]; Patient Health Questionnaire-9 (PHQ-9) [7] [10]; and Kessler-6 Psychological Distress Scale (K6) [6].

\section{Social Factors of Community-Dwelling Elderly People}

The following 4 factors related to social aspects of community-dwelling elderly people were perceived: neighborhood social cohesion, social capital, neighborhood environment, and social support. Neighborhood social cohesion was addressed in 8 studies [6]-[12] [15]. Social capital was addressed in 3 studies [5]

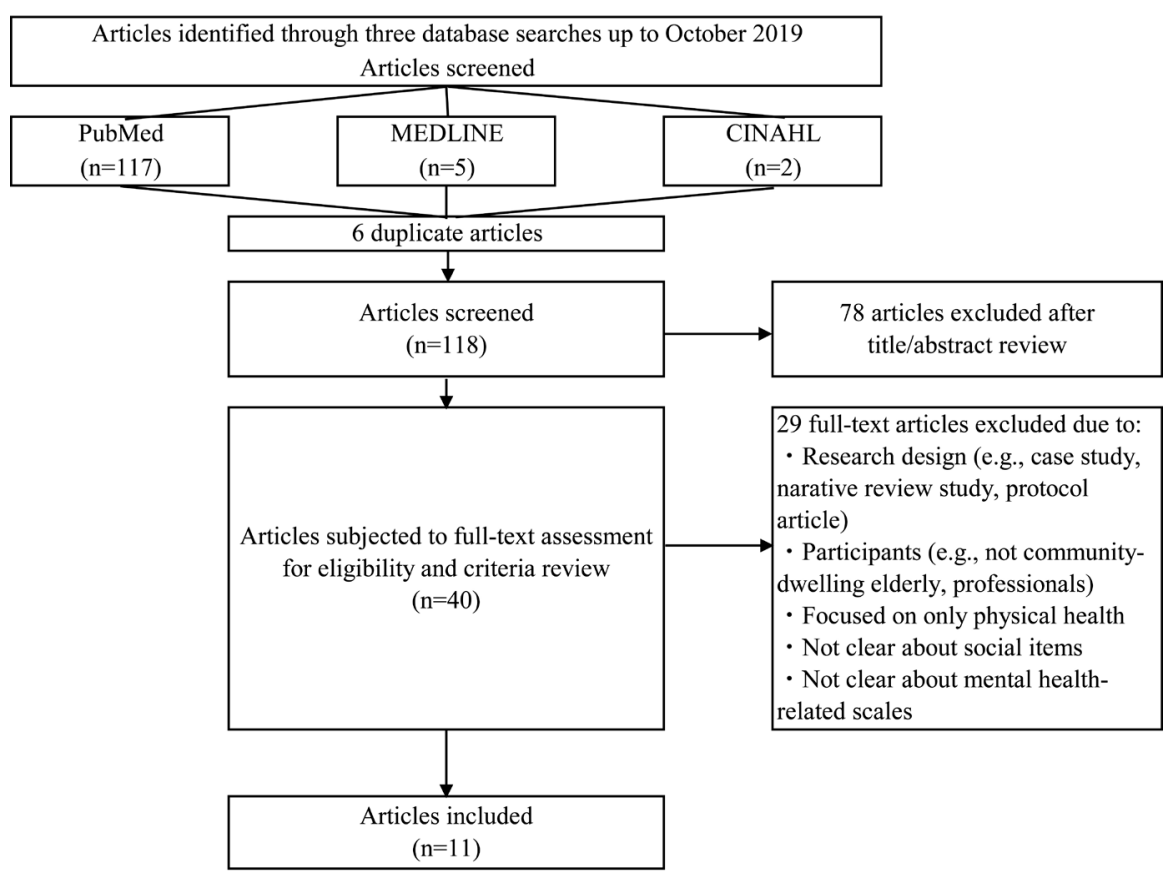

Figure 1. Flowchart of literature search. 


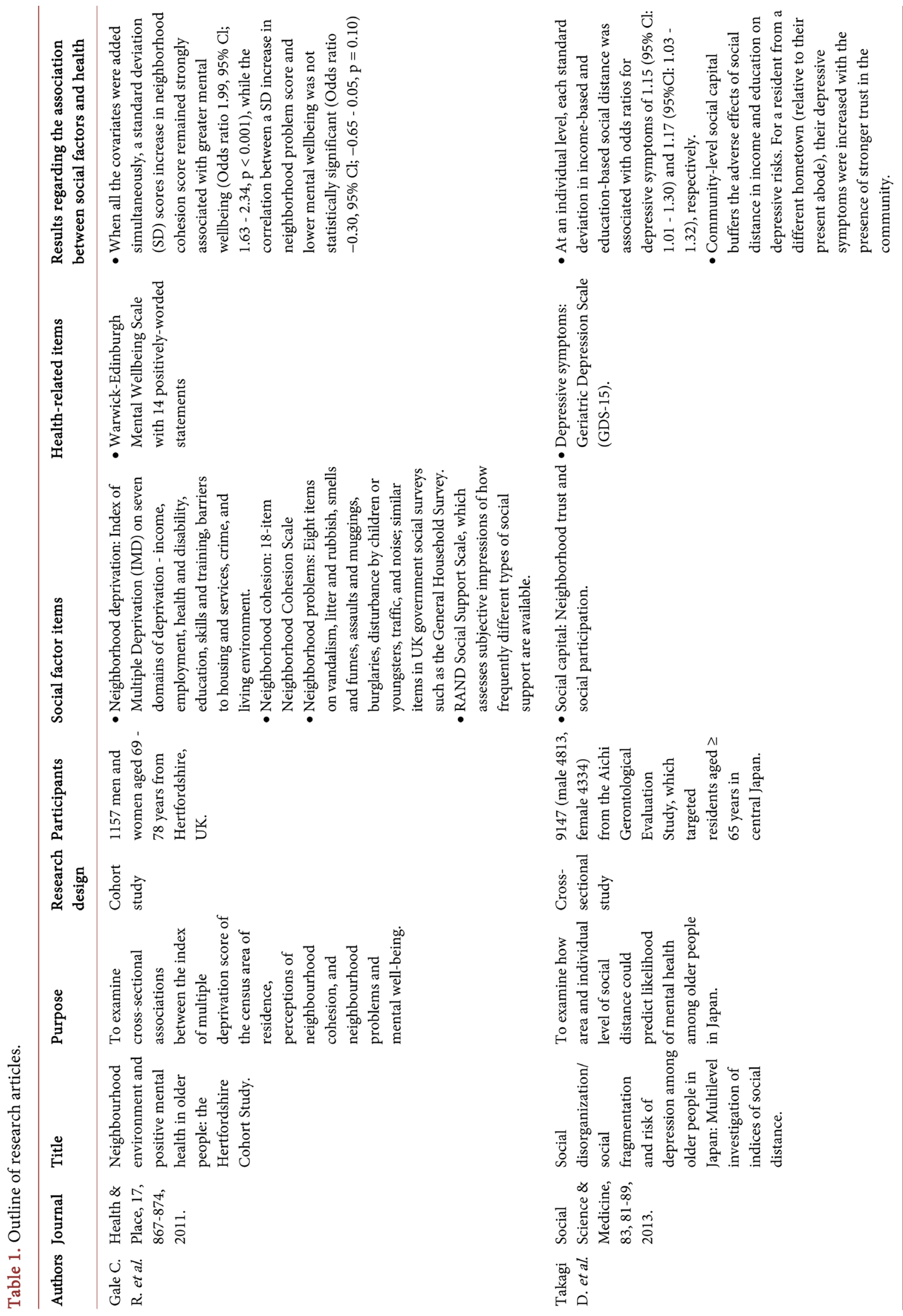




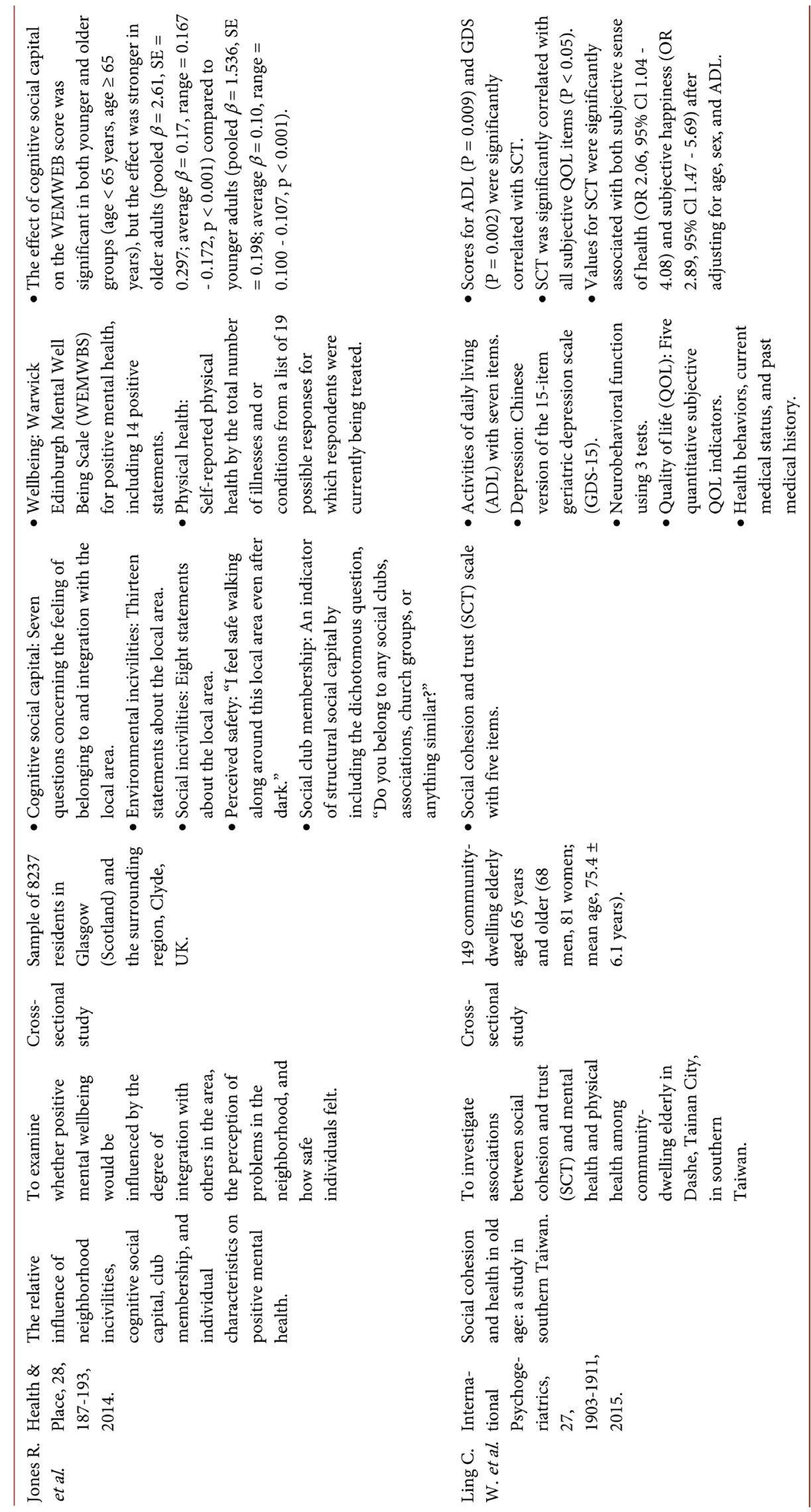




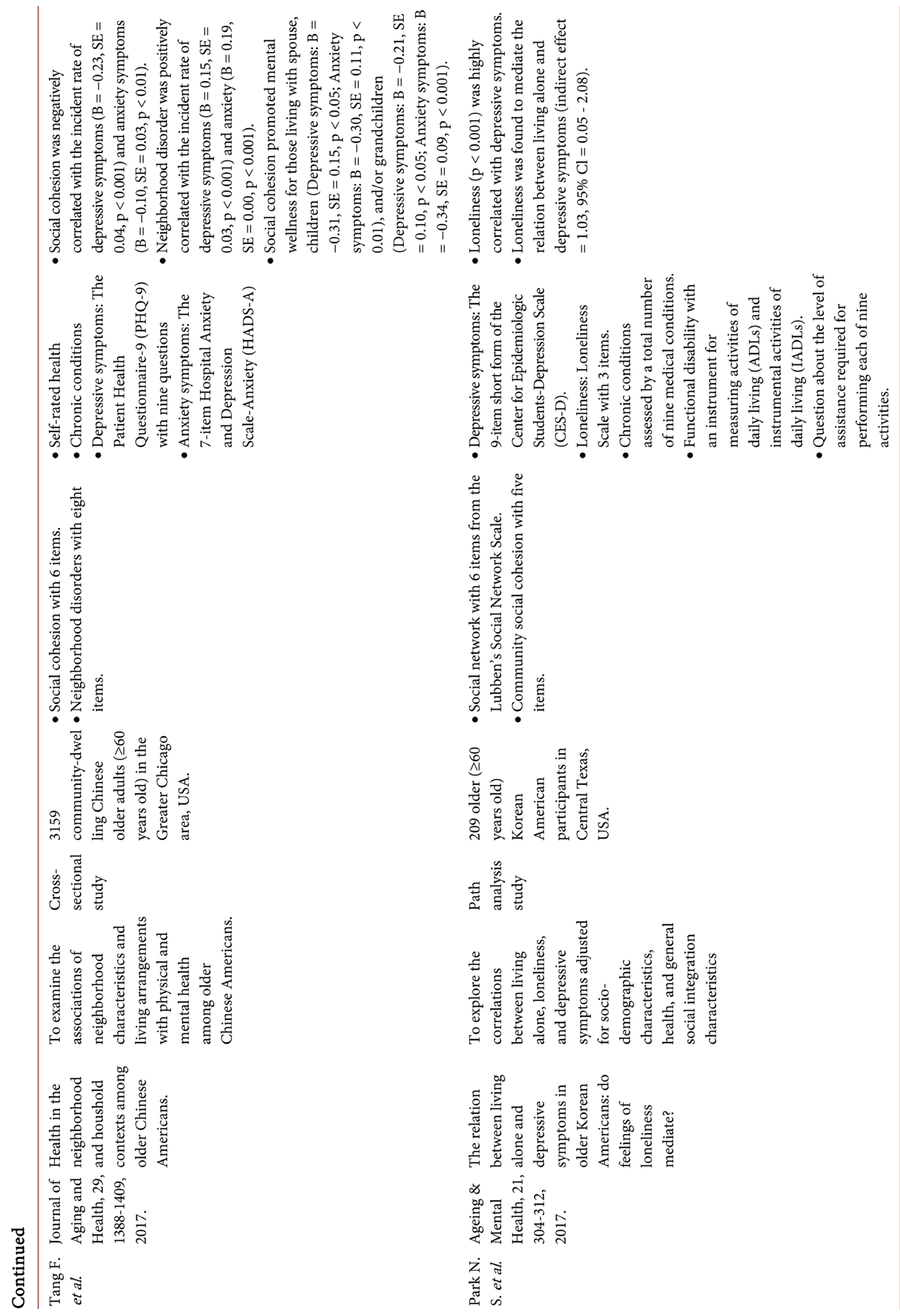




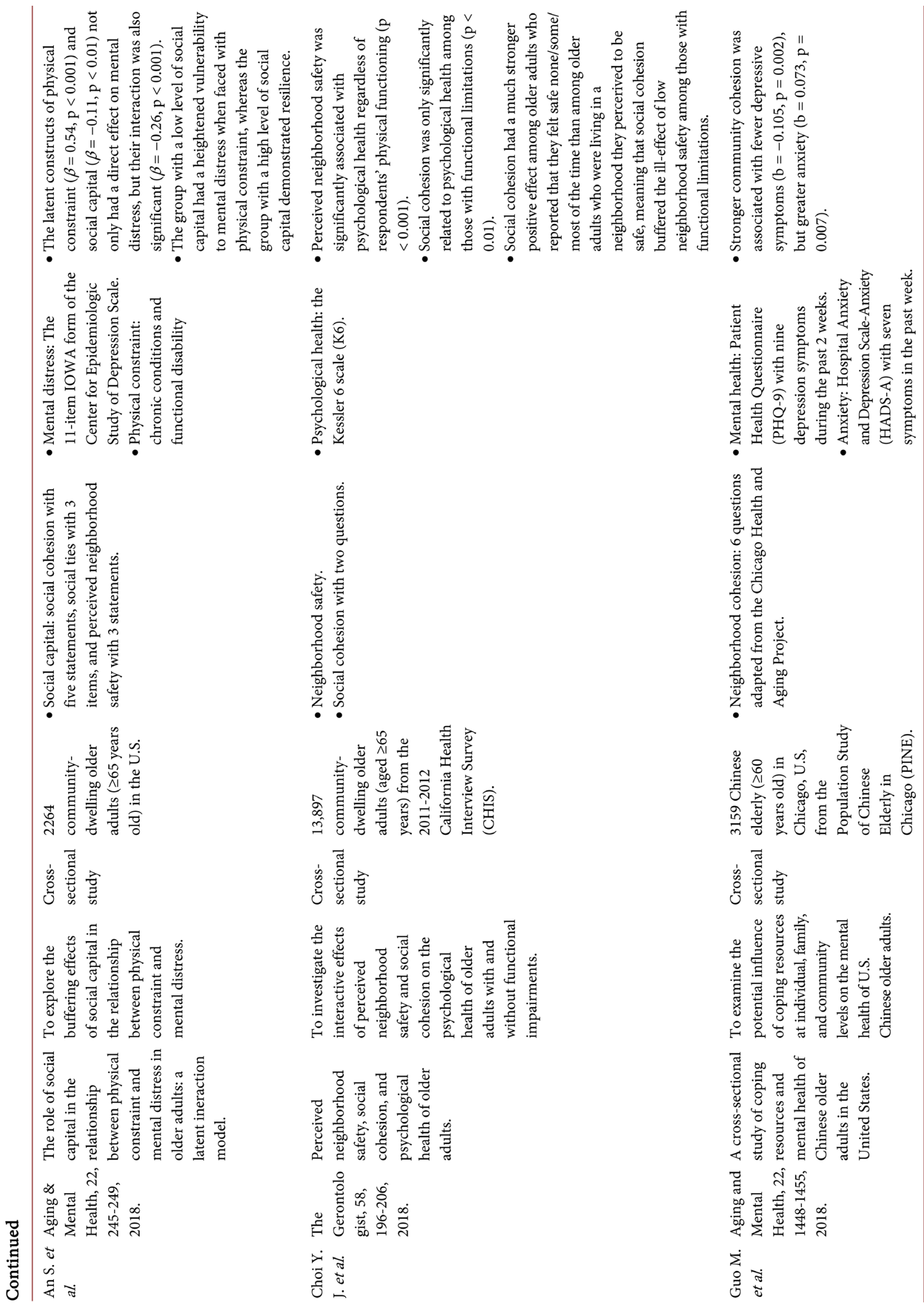




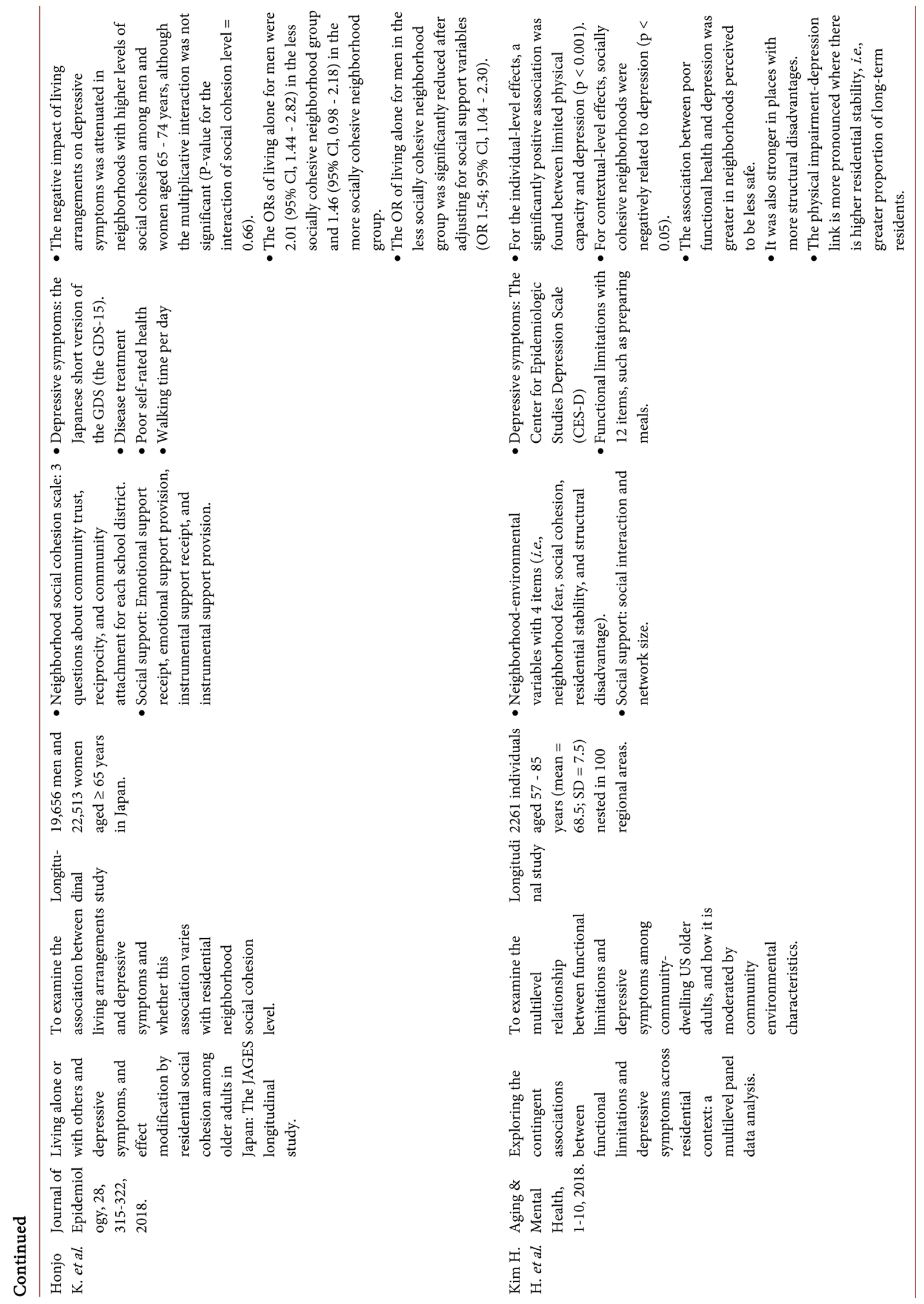


[13] [14]. Neighborhood environment was addressed in 5 studies [6] [9] [10] [13] [15], with items such as neighborhood fear, perceived safety, and neighborhood disorders. Social support was addressed in 3 studies [9] [11] [15] and included items such as emotional support, instrumental support, and social network.

Social Factors and Mental Health of Community-Dwelling Elderly People

The associations between social factors (e.g. neighborhood social cohesion, social capital, neighborhood environment, and social support) and mental health of community-dwelling elderly people were examined.

In neighborhood social cohesion and mental health, a significant association was reported in 6 studies [6] [7] [9] [10] [12] [15]. Gale et al. (2011) found that neighborhood social cohesion was associated with mental health by using the mental health-related scale, WEMWBS ( $\mathrm{p}<0.001$ ). Ling et al. (2015) reported that social cohesion and trust (SCT) was significantly correlated with geriatric depression by using mental health-related scale, GDS-15 ( $\mathrm{P}=0.002)$. Tang et al. (2017) reported that social cohesion was negatively related to incident rates of depressive symptoms by using mental health-related scale, PHQ-9 ( $\mathrm{p}<$ 0.001 ), and anxiety symptoms by using mental health-related scale, HADS-A $(\mathrm{p}<0.01)$. Guo et al. (2018) reported that stronger community cohesion was associated with fewer depressive symptoms by using mental health-related scale, PHQ-9 $(\mathrm{p}=0.002)$, and greater anxiety by using mental health-related scale, HADS-A ( $\mathrm{p}=0.007)$. Choi et al. (2018) reported that social cohesion was significantly related to psychological health by using mental health-related scale, K6 ( $\mathrm{p}<0.01$ ). Kim et al. (2018) reported that socially cohesive neighborhoods were negatively related to depression by using mental health-related scale, CES-D ( $\mathrm{p}<0.05)$.

In social capital and mental health, a significant association was reported in 3 articles [5] [13] [14]. An et al. (2018) reported that social capital had a direct effect on mental distress by using mental health-related scale, CES-D $(\mathrm{p}<0.01)$. Jones et al. (2014) reported that the effect of cognitive social capital on mental health by using WEMWEB was significant $(\mathrm{p}<0.001)$. Takagi et al. (2013) reported that community-level social capital buffered the adverse significant effects of social distance in income and education on depressive risks by using mental health-related scale, GDS-15.

In neighborhood environment and mental health, a significant association was reported in 3 articles [6] [10] [13]. Choi et al. (2018) reported that perceived neighborhood safety was significantly associated with psychological health by using mental health-related scale, K6 ( $\mathrm{p}<0.001)$. Tang et al. (2017) reported that neighborhood disorder was positively associated with incident rate of depressive symptoms by using mental health-related scale, PHQ-9 ( $\mathrm{p}<0.001)$. Jones et al. (2014) reported that higher levels of perceived safety were associated with higher levels of positive wellbeing by using mental health-related scale, WEMWEB ( $\mathrm{p}<$ $0.001)$. 


\section{Discussion}

The composition of the Japanese population continues to change with increasing growth in the elderly portion of the population and declining birth rates, and further decline in the overall population is expected. The Japanese government is currently promoting the development of an integrated community-based care system that takes into consideration the characteristics of residential community in each municipality by 2025 . In the previous literature review studies [2] [3], it was suggested that it might be meaningful to consider social factors, as well as the mental health of elderly people in order to develop effective and sustainable community-based care, considering the characteristics of each community. However, it seemed not to be clear what kinds of social factors and mental health-related scales as outcome measurements should be included in a research. Therefore, in the present study, through the literature review of 11 studies, the social factors and mental health-related scales were perceived, and social factors and mental health of community-dwelling elderly people were considered as outcome measurements for an effective community-based care, with one of the key factors being social cohesion.

4 social factors; neighborhood social cohesion, social capital, neighborhood environment, and social support, were perceived in the present study. It is said that neighborhoods (or residential areas more broadly) have emerged as a potentially relevant group or context because they clearly possess both physical and social attributes which could plausibly affect the health of individuals [16]. The perceived social factors in the present study seemed to include community-level. The findings may suggest the potential usefulness of social factors with regard to determining outcome measures for effective and sustainable integrated community-based care for the elderly, considering the health of the whole population. However, it was suggested that social capital was defined as the combined constructs of community participation and personal social cohesion [17]. For a further investigation, the perceived each social factor in the present study will need to be defined more clearly, in order to determine outcome measurements. And, in the present study, 6 mental health-related scales to assess mental health, depression, and anxiety of the elderly were perceived. For a further investigation, it will need to be considered what kind of mental health-related scale should be used as an outcome measures for an effective community-based care. And, the perceived social factors (e.g. neighborhood social cohesion, social capital, social environment, and social support) seemed to influence mental health of community-dwelling elderly people. Therefore, in the development of effective community-based care, it may be expected to be focused on social aspects to lead to promoting mental health of the elderly.

It was suggested that many studies around the world have shown that living in deprived areas is associated with poorer health, results are often based on a limited number of environmental features and/or aggregated deprivation measures [18]. It will be more important to pile the research data globally by using 
more defined measurements related to social aspects and mental health of the elderly.

\section{Limitation}

There are two points in the limitations of the present study. First, it would not say that 4 perceived social factors in the present study could be defined clearly, because it was not made sure whether the perceived each social factor was statistically independent or not. Second, it would be not enough to show that associations between the social factors and mental health of community-dwelling elderly might be true significant, because of the narrative review, not meta-analysis.

\section{Conclusions}

In the present study, the conclusions through the narrative 11 literature reviews are as follows:

1) As social aspects, 4 social factors; neighborhood social cohesion, social capital, neighborhood environment, and social support, were perceived with regard to determining outcome measures for effective and sustainable integrated community-based care.

2) It was suggested that the perceived social factors significantly associated with the mental health of community-dwelling elderly people. In the development of effective community-based care, it may be expected to be focused on social aspects to lead to promoting mental health of the elderly.

\section{Conflicts of Interest}

The author declares no conflicts of interest regarding the publication of this paper.

\section{References}

[1] Health and Welfare Statistics Association (2019) Kokumin eisei no doukou [Tendency about People's Health]. Journal of Health and Welfare Statistics, 66, 187.

[2] Imaiso, J. (2018) Outcome of Integrated Community Care Interventions for Frail Elderly People: A Literature Review. Health, 10, 1120-1131. https://doi.org/10.4236/health.2018.108085

[3] Imaiso, J. (2019) Significant Differences in Elderly Health between Urban and Rural Communities: A Literature Review. Health, 11, 567-577. https://doi.org/10.4236/health.2019.115048

[4] Fonseca, X., Lukosch, S. and Brazier, F. (2019) Social Cohesion Revisited: A New Definition and How to Characterize It. The European Journal of Social Science Research, 32, 231-253. https://doi.org/10.1080/13511610.2018.1497480

[5] An, S. and Jang, Y. (2018) The Role of Social Capital in the Relationship between Physical Constraint and Mental Distress in Older Adults: A Latent Interaction Model. Aging and Mental Health, 22, 245-249. https://doi.org/10.1080/13607863.2016.1247431

[6] Choi, Y.J. and Matz-Costa, C. (2018) Perceived Neighborhood Safety, Social Cohe- 
sion, and Psychological Health of Older Adults. The Gerontologist, 58, 196-206. https://doi.org/10.1093/geront/gny021

[7] Guo, M., Steinberg, N.S., Dong, X. and Tiwari, A. (2018) A Cross-Sectional Study of Coping Resources and Mental Health of Chinese Older Adults in the United States. Aging and Mental Health, 22, 1448-1455. https://doi.org/10.1080/13607863.2017.1364345

[8] Honjo, K., Tani, Y., Saito, M., Sasaki, Y., Kondo, K., Kawachi, I., et al. (2018) Living Alone or with Others and Depressive Symptoms, and Effect Modification by Residential Social Cohesion among Older Adults in Japan: The JAGES Longitudinal Study. Journal of Epidemiology, 28, 315-322. https://doi.org/10.2188/jea.JE20170065

[9] Kim, H.H. and Youm, Y. (2018) Exploring the Contigent Associations between Functional Limitations and Depressive Symptoms across Residential Context: A Multilevel Panel Data Analysis. Aging and Mental Health, 24, 92-102.

[10] Tang, F., Xu, L., Chi, I. and Dong, X. (2017) Health in the Neighborhood and Household Contexts among Older Chinese Americans. Journal of Aging and Health, 29, 1388-1409. https://doi.org/10.1177/0898264316661829

[11] Park, N.S., Jang, Y., Lee, B.S. and Chiriboga, D.A. (2017) The Relation between Living Alone and Depressive Symptoms in Older Korean Americans: Do Feeling of Loneliness Mediate? Aging and Mental Health, 21, 304-312. https://doi.org/10.1080/13607863.2015.1099035

[12] Ling, C.W., Okumiya, K., Wada, T., Sakamoto, R., Imai, H., Ishimoto, Y., et al. (2015) Social Cohesion and Health in Old Age: A Study in Southern Taiwan. International Psychogeriatrics, 27, 1903-1911. https://doi.org/10.1017/S1041610214002907

[13] Jones, R., Heim, D., Hunter, S. and Ellaway, A. (2014) The Relative Influence of Neighbourhood Incivilities, Cognitive Social Capital, Club Membership and Individual Characteristics on Positive Mental Health. Health and Place, 28, 187-193. https://doi.org/10.1016/j.healthplace.2014.04.006

[14] Takagi, D., Kondo, K., Kondo, N. Cable, N., Ikeda, K. and Kawauchi, I. (2013) Social Disorganization/Social Fragmentation and Risk of Depression among Older People in Japan: Multilevel Investigation of Indices of Social Distance. Social Science and Medicine, 83, 81-89. https://doi.org/10.1016/j.socscimed.2013.01.001

[15] Gale, C.R., Dennison, E.M., Cooper, C. and Sayer, A.A. (2011) Neighbourhood Environment and Positive Mental Health in Older People: The Hertfordshire Cohort Study. Health and Place, 17, 867-874.

https://doi.org/10.1016/j.healthplace.2011.05.003

[16] Diez-Roux, A.V. (2007) Neighborhoods and Health: Where Are We and Do We Go from Here? Environment résidential et santé: État de la question et perspectives pour le future. Revue d' Epidémiologie et de Santé Publique, 55, 13-21. https://doi.org/10.1016/j.respe.2006.12.003

[17] Berry, H.L. and Welsh, J.A. (2010) Social Capital and Health in Australia: An Overview from the Household, Income and Labour Dynamics in Australia Survey. Social Science and Medicine, 70, 588-596. https://doi.org/10.1016/j.socscimed.2009.10.012

[18] Putrik, P., Vries, N.K., Mujakovie, S., Amelsvoort, L., Kant, I., Kunst, A.E., et al. (2015) Living Environment Matters: Relationship between Neighborhood Characteristics and Health of the Residents in a Dutch Municipality. Journal of Community Health, 40, 47-56. https://doi.org/10.1007/s10900-014-9894-y 